\title{
Evaluating Techniques for Estimating Post-Breeding-Season Age Ratios for American Woodcock
}

KYLE O. DALY, ${ }^{1,2}$ Minnesota Cooperative Fish and Wildlife Research Unit, Department of Fisheries, Wildlife, and Conservation Biology, University of Minnesota, St. Paul, MN 55108, USA

DAVID E. ANDERSEN, U.S. Geological Survey, Minnesota Cooperative Fish and Wildlife Research Unit, University of Minnesota, St. Paul, MN 55108, USA

WAYNE L. BRININGER, U.S. Fish and Wildlife Service, Tamarac National Wildlife Refuge, 35704 County Road 26 Rochert, MN 56578, USA

THOMAS R. COOPER, U.S. Fish and Wildlife Service, Migratory Bird Program, 5600 American Boulevard W, Suite 99o, Bloomington, MN 55437, USA

\begin{abstract}
Estimating American woodcock (Scolopax minor; hereafter, woodcock) vital rates at a landscape scale requires considerable effort and expense, but provides a means of assessing population response to management. Age ratios derived from capturing woodcock (e.g., age ratios derived from mist-netting or night-lighting) during late summer may be useful proxies of local production, and require much less effort and expense to obtain than estimating local production directly. To assess whether such age ratios were similar to estimates of production derived from estimating vital rates, we estimated post-breeding-season age ratios (juveniles/adult female) at a habitat-management demonstration area in west-central Minnesota using radio telemetry and nest monitoring, and by capturing woodcock using mist nets and night-lighting. In 2011 and 2012 we radio-marked and tracked 41 adult female and 73 juvenile woodcock and monitored 51 broods and 48 nests; we used the collected data to inform population models and derive post-breeding-season age ratios. In July of 2011 and 2012, we captured 204 woodcock using mist nets by exploiting crepuscular movements from diurnal feeding cover to roosting fields and 69 woodcock via night-lighting on nocturnal roosting fields. Estimates of age ratios derived from our population model were 1.07 (95\% CI: $0.27-2.45$ ) in 2011 and 2.59 (95\% CI: 1.04 - 4.95) in 2012. We attribute the higher point estimate of age ratio in 2012 to higher nest and juvenile survival rates during that year. Age ratios from mist-netting were 3.82 (95\% CI: $1.99-7.13$ ) in 2011 and 2.37 (95\% CI: $1.43-3.73$ ) in 2012 and from night-lighting were 1.62 (95\% CI: $0.69-3.28$ ) in 2011 and 0.42 ( $95 \%$ CI: $0.06-1.00$ ) in 2012. Age ratio point estimates derived from mist-netting and night-lighting varied considerably between years, with neither method providing a ratio similar to point estimates derived from our population model. The only statistically significant difference (based on 95\% CIs) between any of our age ratio estimates was for night-lighting in 2012, with that estimate being lower than the estimates from mist-netting and our population model. Based on these results, age ratios of production of young derived from mist-netting and might-lighting in late summer may not reflect local production, and may be influenced by both local and landscape-scale movements of woodcock following the breeding season. We conclude that without further evaluation of factors that affect post-breeding-season age ratios of local woodcock populations, age ratios derived from mist-netting and night-lighting in summer may not be useful proxies of local recruitment.
\end{abstract}

Proceedings of the American Woodcock Symposium 11: 35-44

KEY WORDS: American woodcock, post-breeding-season age ratio, Minnesota, Scolopax minor, survival

1 email: kyle_daly@fws.gov

2 current address: U.S. Fish and Wildlife Service, Wildlife and Sport Fish Restoration Program,

5600 American Boulevard W, Suite 990, Bloomington, MN 55437, USA 
American woodcock (Scolopax minor; hereafter, woodcock) have experienced long-term population declines across portions of their breeding range, based on the spring American Woodcock Singing-Ground Survey (Seamans and Rau 2017). Wing-collection surveys also have indicated a decline in woodcock productivity, especially in the Central Management Region (Seamans and Rau 2017). These declines have been speculated to be due to loss or alteration of young forest cover types that support woodcock reproduction (Gregg 1984, Dwyer et al. 1988, Sauer and Bortner 1991, Kelley et al. 2008). In response to these apparent declines in woodcock abundance and productivity, a system of woodcock habitat-demonstration areas is being developed throughout the woodcock breeding range where specific Best Management Practices (BMPs) are applied with the goal of stabilizing and ultimately increasing woodcock populations (Wildlife Management Institute 2010). These demonstration areas are meant to promote young forest management on public and private lands, and to increase the abundance and distribution of young forest cover types. Woodcock use managed areas (e.g., Hale and Gregg 1976, Wildlife Management Institute 2009), but it is unknown whether apparent increases in local woodcock abundance (Dwyer et al. 1988) are a result of movement of woodcock into these areas, of these landscapes increasing population growth rates, or of a combination of these and other factors. Part of the difficulty in assessing the effects of management for woodcock at a landscape scale is that there are no established methods to efficiently estimate production of young (e.g., as reflected in post-breeding-season age ratios).

An index of woodcock production (juveniles/adult female in the harvest) is reported annually for the Eastern and Central Management Regions and by state through a wing collection survey (Seamans and Rau 2017). These indices are used to assess both short- and long-term trends for woodcock productivity at broad spatial scales. Estimates of post-breeding-season age ratios at a demonstration-area scale ( 200-80o ha), using the same metric as wing collection surveys, also can be used to assess whether BMPs applied at demonstration areas result in increased woodcock production. However, estimating post-breeding-season age ratios with methods that rely on estimates of local woodcock vital rates (e.g., estimating survival via telemetry and nest success via nest monitoring) can be expensive and require in-depth field studies. Age ratios derived from capturing woodcock during late summer (e.g., capture via night-lighting or mist-netting, see below) may provide an alternative to deriving post-breeding-season age ratios based on estimates of woodcock vital rates and may be more cost- and effort-efficient. However, it is not known whether post-breeding-season age ratios reflect local productivity at the scale of demonstration areas.
Our objectives were to (1) estimate woodcock post-breeding-season age ratios at a demonstration-area scale by monitoring nests and radio-monitoring adult females and juveniles as a measure of local woodcock production, (2) derive and compare age ratios from mist-netting and night-lighting during late summer, and (3) evaluate whether model-based estimates of age ratios and those derived from mist-netting and night-lighting were similar (and therefore might be useful as proxies of local production). Similar age ratio estimates between model-based and mist-netting and night-lighting would suggest that less costly and labor-intensive methods could be used to monitor production at the demonstration-area scale. Dissimilar age ratios would suggest bias in 1 or more of these methods, or biological factors (e.g., local or landscape-scale woodcock movements during summer) that changed age ratios following when young fledged.

\section{Study Area}

As part of a larger study of woodcock population ecology, we estimated post-breeding-season age ratios in late summer in 2011 and 2012 at Tamarac National Wildlife Refuge (NWR) near Rochert, Minnesota, USA (47.0 N, -95.7 E). Tamarac NWR is a woodcock habitat demonstration area, lies in the glacial lake country of west-central Minnesota in Becker County, and encompasses 17,296 ha of mostly forested lands intermingled with lakes, rivers, marshes, shrub swamps, and tallgrass prairie. Tamarac NWR is located in the transition zone between coniferous forest, northern hardwood forest, and tallgrass prairie. Sixty percent of the refuge is forested, with dominant tree species of aspen (Populus spp.), jack pine (Pinus banksiana), red pine (P. resinosa), balsam fir (Abies balsamea), paper birch (Betula papyrifera), red oak (Quercus rubra), white oak (Q. alba), sugar maple (Acer saccharum), and basswood (Tilia americana). A substantial portion of the refuge is maintained in early successional forest cover, using timber harvest and prescribed fire, to provide habitat for woodcock, golden-winged warblers (Vermivora chrysoptera), and other migratory birds that utilize young forest cover types.

\section{METHODS}

\section{ESTIMATING POST-BREEDING-SEASON AGE RATIOS FROM RADIO TELEMETRY AND NEST MONITORING}

To estimate post-breeding-season age ratios derived from local woodcock vital rates, we monitored survival of woodcock and woodcock nests. In early spring of 2011 and 2012, we used mist nets (Avinet 38-mm black polyester nets, 4 pockets, $2.6 \mathrm{~m}$ high, 6 and $9 \mathrm{~m}$ wide; Avinet, Inc., Dryden, NY) to capture woodcock during dusk (approximately 1900 to 2300 CDT) when woodcock leave diurnal areas to roost or feed (Sheldon 1971). We determined sex of all woodcock captured based on plumage characteris- 
tics (Martin 1964), and radio-marked adult female woodcock using a glue-on backpack-style harness that in combination with the transmitter was $\leq 3 \%$ of their total mass (4.5 g, model A5410, Advanced Telemetry Systems, Isanti, MN; McAuley et al. 1993a, b). We relocated radio-marked female woodcock 5-7 days per week throughout the breeding season (April-June) and into July, following the nesting period. We lost radio contact with some females when they traveled long distances, out of the range of our receiving equipment and monitoring protocol. We continued searching for these females throughout our field season and, if we did not relocate them, censored them in our analyses at the time of their last known location. When we relocated a radio-marked adult female, we assessed its status (i.e., alive or dead) and, if it was dead, assessed the cause of death. If depredated, we attempted to ascertain the source of predation (mammalian or avian) using methods described by McAuley et al. (2005).

We found woodcock nests using trained pointing dogs (Ammann 1977, McAuley et al. 1993a) and radio telemetry of incubating radio-marked adult females. We visited each nest every 2-3 days and assessed the status of the nest as active, depredated, abandoned, or successful. If the female was not present at the nest or flushed during our visit, we floated all eggs in ambient-temperature water to estimate nest age and initiation date (Ammann 1974). We considered nests to be active during periods when the adult female was either laying or incubating eggs, or if a female was incubating at a subsequent visit. We categorized nests as depredated if eggs were broken or absent prior to the estimated hatch date. We categorized nests as abandoned if the female was not observed incubating for 2 consecutive visits. We categorized nests as successful if $\geq 1$ egg exhibited signs of having hatched (i.e., was in or close to the nest bowl with a longitudinal split).

We assessed the status of juveniles in broods of radiomarked adult females and also radio-marked a sample of juveniles within broods of radio-marked adult females. We used trained pointing dogs to find broods of unmarked adult females (Mendall 1938; Ammann 1974, 1977), and captured and radio-marked juveniles in those broods. We custom fit a collar-type micro-transmitter (BD-2NC or BD-2C, Holohil Systems Ltd., Carp, ON and custom transmitters made by Blackburn Transmitters, Nacogdoches, TX) with a whip antenna to captured juvenile woodcock. All transmitters were $\leq 3 \%$ of the bird's mass (BD-2NC transmitters weighed about $0.6 \mathrm{~g}, \mathrm{BD}-2 \mathrm{C}$ transmitters weighed about $1.6 \mathrm{~g}$, and Blackburn transmitters weighed about $0.4 \mathrm{~g}$ ) and we attached transmitters to juvenile woodcock with an elastic collar designed to expand as the juvenile woodcock grew. We positioned transmitters at the base of a juvenile woodcock's neck with the transmitter antenna lying down the juvenile's back. Daly et al. (2015) demonstrated that this attachment method had no influ- ence on subsequent survival rate. Transmitter lifespan was about 21 days (17-30 day range) for $\mathrm{BD}-2 \mathrm{NC}$ transmitters, 63 days (49-77 day range) for $\mathrm{BD}-2 \mathrm{C}$ transmitters, and 28 days (24-32 day range) for Blackburn transmitters.

We radio-marked 1-4 juveniles per brood and monitored both marked and unmarked individuals within a brood after locating radio-marked juveniles. We considered juveniles within the same brood as independent experimental units in our study because Daly et al. (2015) found no evidence of dependence among juveniles in the same broods using Winterstein's (1992) second Chi-square goodness-of-fit test $\left(\chi^{2}=17.2, P=0.99\right.$; data reanalyzed using Winterstein's third Chi-square goodness-of-fit test in Breeding Season Survival of Woodcock, Appendix II, page 62). The survival of a juvenile woodcock was thus not statistically linked to the survival of broodmates, making it appropriate to treat each juvenile within a brood as an individual sample. We located broods 4-7 days per week by tracking either the adult female or juvenile(s). We assessed status (alive or dead) of juveniles, counted both marked and unmarked juveniles to document brood size, and assumed juveniles were dead if previously observed but subsequently not detected. We counted the number of individuals in the brood by tracking the radio-marked woodcock(s) to a distance of about $5 \mathrm{~m}$ and then encircling the brood until we were certain all brood members were counted. Beginning about 15 days after hatch, entire broods often flushed at our approach, which afforded us the opportunity to determine total brood size. Beyond 15 days post-hatch, the probability of detecting all members of the brood diminished. Because we were not able to accurately determine the status of unmarked juveniles after brood breakup, we right-censored unmarked juveniles at 24 days old, which was earliest we observed brood breakup.

We recorded the number of days post-transmitter deployment on females and juveniles to accurately censor individuals if radio transmitters failed prematurely. We assumed radios failed if they performed irregularly and there was no other indication an individual had died. We also assumed radios failed if they were nearing the end of their projected battery life and we subsequently received no additional signals from transmitters. We right-censored individuals in both of these circumstances, assuming the individual survived until transmitter failure (e.g., Korschgen et al. 1996).

We estimated survival rates of adult females, nests, and juveniles using the Kaplan-Meier with staggered entry estimator (Pollock et al. 1989, Nur et al. 2004) in the KMsurv package in Program R (version 2.15.2, R Core Team, 2012). We used these survival rate estimates to construct a model of the woodcock population in our study area and to derive post-breeding-season age ratio estimates in 2011 and 2012, which were the years we also derived age ratios via mist-netting and night-lighting. For the purposes of our 
model, we estimated adult female survival rate for a 122 day period (1 April - 31 July). We estimated nest survival rate for a 24-day period that included 3 days for egg laying and 21 days for incubation. Because the ages of the nests were known, we entered all nests into our analysis when they were initiated. We estimated juvenile survival rate for a 92-day period (1 May - 31 July).

We used estimates of period survival rate to estimate the number of female woodcock surviving the breeding season, and the number of juvenile woodcock produced and surviving through the end of the breeding season (31 July). We created an arbitrary population of 10,000 adult females and randomly selected values within our 95\% CI for Kaplan-Meier period survival rate estimates of adult females to inform our population model for adult females surviving until the end of the breeding season,

Adult females surviving the breeding season

$$
=10,000 * \mathrm{FS}
$$

where FS is a randomly selected value of adult female survival rate during the breeding season.

To supplement estimates of survival rates on our study area, we used published estimates of renesting rate, clutch sizes, and hatch rate to model the woodcock population on our study area. Woodcock are known to renest up to one time during a breeding season following a failed nest or if they lose a brood <11 days old (McAuley et al. 1990). Because we captured females into the nesting portion of the breeding season, and the time from nest or brood failure to renesting can be as short as 4-5 days ( $\mathrm{McAu}-$ ley et al. 1990, also observed during this study), we were unable to assess whether the majority of nests in our study were first nests or renesting attempts. We therefore assumed the same survival probability for first nests and renests. Because of a high renesting probability for woodcock ( 93\%, McAuley et al. 1990), we prescribed a second nest to all woodcock in our population model that lost a first nest. The clutch size of first nesting attempts averages 4 eggs, whereas the clutch for renesting attempts is usually 3 eggs per nest, and both first nesting and renesting attempts have high hatch rates ( 0.95; McAuley et al. 1990).

We initiated our model with all 10,000 adult female woodcock initiating a nest to calculate the number of juveniles produced. We applied the Kaplan-Meier estimate of nest survival rate to all nests and assumed that clutch size was 4 eggs in first nesting attempts and 3 eggs for renesting attempts. We assumed that if the nest was successful (i.e., $\geq 1$ egg hatched), the hatch rate of eggs was 0.95 . We estimated nest productivity (i.e., number of juveniles hatching; NP) as,

$$
\begin{gathered}
\mathrm{N} P=\left(10,00{ }^{\star} 44^{\star} \mathrm{HR}{ }^{\star} \mathrm{NS}\right)+ \\
\left(\left[10,000-\left(10,00{ }^{*} \mathrm{NS}\right)\right]^{\star} 3^{\star} \mathrm{HR}^{\star} \mathrm{NS}\right)
\end{gathered}
$$

where HR is hatch rate, and NS is a randomly selected value for nest survival within our 95\% CI based on KaplanMeier estimates. We recalculated this estimate 1,00o times to derive a $95 \%$ confidence interval by excluding $2.5 \%$ of the highest and lowest point estimates.

From these estimates of nest productivity we calculated the number of juveniles that survived through the breeding season. We applied the Kaplan-Meier estimate of juvenile survival rate to the resulting number of juveniles to calculate the number of juveniles that were produced in 2011 and 2012, separately. We estimated the number of juveniles surviving to the end of the breeding season as

$$
\text { Population of Juveniles }=\mathrm{NP}{ }^{\star} \mathrm{JS}
$$

where JS is a randomly selected value for juvenile period survival rate within our 95\% CI around Kaplan-Meier survival-rate estimates.

We randomly selected period-survival-rate estimates with replacement for adult female, nest, and juvenile survival rates and conducted 1,000 iterations of calculations of population sizes of adult females and juveniles. For each iteration, we calculated post-breeding-season age ratios by dividing the number of juveniles that were produced and survived the breeding season by the number of adult females that survived the breeding season. From these 1,000 estimates of post-breeding-season age ratio, we calculated the mean, and the $95 \% \mathrm{CI}$ as the interval that excluded $2.5 \%$ of estimates on either end of the distribution.

\section{ESTIMATING POST-BREEDING-SEASON AGE RATIOS FROM MIST-NETTING AND NIGHT-LIGHTING}

We captured woodcock on summer roost fields starting in early July and concluding in early August in both 2011 and 2012 (Dwyer et al. 1988). We used mist nets to capture woodcock during crepuscular movements at dusk (approximately 1900 to 2300 CDT) and calculated net nights as the sum of the total number of mist nets set per night. We captured woodcock via night-lighting in known woodcock roosting areas (Dwyer et al. 1988) following the period of crepuscular movement. Each night we had a single person shining a spotlight (Cabela's 35-Watt HID spotlight, 3,000 lumens and Cyclops Sirius 500 spotlights, 500 lumens; Cabela's, Sydney, NE) and 1-2 people attempting to capture woodcock with long-handled nets. We assigned age (hatch year or after hatch year) and sex to all captured woodcock using body measurements and feather characteristics (Martin 1964, Sepik 1994).

We used bootstrapping techniques to resample with replacement for 1,000 iterations from woodcock captured mist-netting and night-lighting in 2011 and 2012 to derive woodcock post-breeding-season age ratios. We estimated the post-breeding-season age ratio for each itera- 
tion and derived the mean and the 95\% CI based on the interval that excluded $2.5 \%$ of estimates on either end of the distribution of the 1,000 estimates we derived from bootstrapping.

We compared the post-breeding-season age ratios derived from our population model to those derived from woodcock captured via mist-netting and night-lighting based on 95\% CIs, where non-overlap of 95\% CIs indicated statistical significance. We also compared the age ratios in our study to those obtained from the wing-collection survey at both statewide and Central Management Regionwide scales (Cooper and Rau 2012, 2013).

Capture and marking protocols were approved by the University of Minnesota Institutional Animal Care and Use Committee under Protocol no.1103A97333.

\section{Results}

\section{POST-BREEDING-SEASON AGE RATIOS FROM}

\section{RADIO TELEMETRY AND NEST MONITORING}

From 9 April to 1 June 2011 and 22 March to 24 May 2012, we captured and radio-marked 41 adult female woodcock (2011: $n=23,2012: n=18)$. We excluded $3(\sim 7 \%)$ radio-marked adult female woodcock in 2011 that we were unable to relocate following radio marking (likely due to transmitter failure or migration) from survival analysis. Of the remaining 38 female woodcock, we right-censored 1 female that became entrapped in its radio transmitter harness in 2012.

From 4 May to 11 June 2011 and from 9 April to 9 June 2012 we monitored 52 woodcock nests (2011: $n=26,2012$ : $n=26)$. We right-censored 1 nest in 2011 and 3 in 2012 . We excluded an additional 3 nest in 2011, 1 because we were not able to relocate it after first detection, and 2 we attributed to abandonment caused by our activities. Of the 45 nests we included in our survival-rate analyses, 21 $(\sim 47 \%)$ failed, 15 were depredated $(71 \%)$, and 6 (29\%) were abandoned by the female.

From 16 May to 29 June 2011 and 20 April to 16 June 2012 we radio-marked 73 (2011: $n=22,2012: n=$ 51) juvenile woodcock from 51 broods (2011: $n=16$, 2012: $n=35$ ). We assigned fates to 134 juvenile woodcock (2011: $n=63$, 2012: $n=71$ ), including fates of 49 marked and 85 unmarked juveniles from 47 broods (2011: $n=23,2012$ : $n=24$ ). We excluded data from 24 marked juvenile woodcock due to problems with transmitter operation and failure (i.e., weak signals where we could not relocate the individual and did not know if the transmitter failed or if the juvenile was depredated).

Survival-rate estimates during the breeding season for adult female woodcock from 1 April to 31 July were 0.616 (95\% CI: $0.427-1.000$ ) in 2011 and 0.695 (95\% CI: $0.427-$ 1.000 ) in 2012 (Table 1). Kaplan-Meier estimates of survival rates for the 24-day laying and incubation period for nests were 0.458 (95\% CI: $0.299-0.696)$ in 2011 and 0.786 (95\%
CI: $0.616-1.000$ ) in 2012 (Table 1). Kaplan-Meier estimates of survival rates for juvenile woodcock for a 91-day period (1 May - 31 July) were 0.191 (95\% CI: $0.083-0.481$ ) in 2011 and 0.401 (95\% CI: $0.253-0.761$ ) in 2012 (Table 1). Our estimates of post-breeding-season age ratios based on survival and reproduction of females and survival of nests and juveniles were 1.07 (95\% CI: $0.27-2.45)$ in 2011 and 2.62 (95\% CI: $1.04-4.95$ ) in 2012 (Table 2).

\section{POST-BREEDING-SEASON AGE RATIOS FROM MIST-NETTING AND NIGHT-LIGHTING}

In 2011, post-breeding-season age ratios varied considerably as a function of capture technique. We captured more woodcock using mist-netting than night-lighting, in part because night-lighting is effective only under very specific conditions (e.g., nights with little ambient light). We spent 16.3 hours mist-netting and 23.5 hours night-lighting between 7 July and 24 July 2011 . We set an average of 9.5 mist nets per night and mist-netting effort totaled 114 trap nights. The capture rate for mist-netting on summer roosting fields was 5.3 woodcock per hour (across the average 9.5 mist nets per night), whereas the capture rate for night-lighting on roosting fields was 1.8 woodcock per hour. We captured 3.50 juveniles per adult female $(n=87)$ via mist-netting and 1.46 juveniles per adult female $(n=$ 42) via night-lighting (Table 2). Age ratios for woodcock captured via mist-netting were 3.82 (95\% CI: 1.99 - 7.13) and 1.62 (95\% CI: $0.69-3.28)$ for night-lighting (Table 2).

Table 1. American woodcock survival rate estimates for adult females, nests, and juveniles from Tamarac National Wildlife Refuge, Rochert, Minnesota, USA, in 2011 and 2012. We calculated survival rate estimates using the Kaplan-Meier method with staggered entry (Pollock et al. 1989) in the KMsurv package in Program $\mathrm{R}$; period survival rates for female woodcock for a 122-day period, for juvenile woodcock for a 92-day period, and for woodcock nests for a 24-day egg-laying and incubation period.

\begin{tabular}{ccc}
\hline Year & $\begin{array}{c}\text { Period survival rate } \\
(\mathbf{9 5 \%} \text { CI })\end{array}$ & $\begin{array}{c}\text { Daily survival rate } \\
(\mathbf{9 5 \%} \text { CI })\end{array}$ \\
\hline $\begin{array}{cc}\text { Females } \\
2011\end{array} \quad 0.616(0.427-1.000)$ & $0.996(0.993-1.000)$ \\
2012 & $0.695(0.427-1.000)$ & $0.997(0.993-1.000)$ \\
\hline Nests $(24$ days $)$ & \\
2011 & $0.458(0.299-0.696)$ & $0.968(0.951-0.985)$ \\
2012 & $0.786(0.616-1.000)$ & $0.990(0.980-1.000)$ \\
\hline Juveniles $(92$ days $)$ & \\
2011 & $0.191(0.083-0.481)$ & $0.982(0.973-0.992)$ \\
2012 & $0.401(0.253-0.761)$ & $0.991(0.985-0.997)$ \\
\hline
\end{tabular}


In 2012, the age ratio of woodcock captured via mist-netting was higher than that for woodcock captured via night-lighting. We spent 40 hours mist-netting and 29 hours night-lighting between 1 July and 30 July 2012, resulting in a capture rate of 2.92 woodcock per hour mist-netting and 0.93 woodcock per hour night-lighting. Trapping effort for mist-netting totaled 220 trap nights with an average of 10.5 mist nets set per night. We captured 2.28 juveniles per adult female $(n=117)$ via mist-netting and 0.38 juveniles per adult female $(n=27)$ via night-lighting (Table 2). Our post-breeding-season age ratios were 2.37 (95\% CI: $1.43-3.73$ ) for mist-netting and 0.42 (95\% CI: $0.06-1.00$ ) for night-lighting (Table 2).

\section{COMPARISON OF POST-BREEDING- SEASON AGE RATIOS}

In both 2011 and 2012, point estimates of age ratios of woodcock captured via mist-netting were higher than those of woodcock captured via night-lighting (Table 2, Fig. 1), but point estimates of age ratios of woodcock captured via both mist-netting and night-lighting were lower in 2012 than in 2011. In contrast, point estimates of age ratio estimates derived from our population model were higher in 2012 than 2011. In 2011, the point estimate of the age ratio of woodcock captured via mist-netting was not similar to the point estimate of the age ratio derived from our population model, but these estimates were similar in 2012. The point estimates of the age ratio of woodcock captured via night-lighting were dissimilar to those derived from our population model in both 2011 and 2012 . Similarly, the magnitude and direction of the difference between point estimates of age ratios derived in 2011 and 2012 were not similar for either mist-netting or night-lighting compared to the difference in age ratios derived from our population model between 2011 and 2012 (Table 2, Fig. 1). The only statistically significant difference between any of our age ratio estimates (based on non-overlapping 95\% CIs) was for night-lighting in 2012, with the estimate being lower than the estimates from mist-netting and our population model (Table 2).

\section{Discussion}

A primary objective of our assessment was to evaluate whether post-breeding-season age ratios of woodcock captured via mist-netting and night-lighting could serve as useful proxies for age ratios derived from a population model informed by estimates of local vital rates. Point estimates of age ratios derived from woodcock captured via mist-netting or night-lighting were not consistently related to point estimates of the post-breeding-season age ratio derived from our population model. However, all of the methods we used produced imprecise estimates of post-breeding-season age ratios, resulting in only 1 instance of a statistically significant difference between estimates derived using different methods (Table 2), and suggesting a need for additional assessments of these methods to estimate local production at the demonstration-area scale. Based on estimates derived from our

Table 2. Post-breeding-season age ratios (juveniles/adult female) derived from capturing American woodcock in summer roosting field via mist-netting and night-lighting, and an estimate from a population model based on estimates of vital rates at Tamarac National Wildlife Refuge, Rochert, Minnesota, USA, in 2011 and 2012. Post-breeding-season age ratios from mist-netting and night-lighting were created using bootstrapping with 1,00o iterations for 2011 and 2012. We calculated post-breeding-season age ratio for each iteration and derived the mean and $95 \% \mathrm{CI}$ based on the distribution of post-breeding-season age ratios (excluding the lowest and highest $2.5 \%$ of estimates). Post-breedingseason age ratio estimates from our population model were informed by Kaplan-Meier survival rate estimates of adult female, nests, and juvenile woodcock. Post-breeding-season age ratios estimates are sample means derived from bootstrapping using 1,00o iterations and $95 \%$ confidence intervals were estimated based on the distribution of estimates of post-breeding-season age ratios.

\begin{tabular}{lcccccc}
\hline Method & $\begin{array}{c}\text { Adult } \\
\text { male }\end{array}$ & $\begin{array}{c}\text { Adult } \\
\text { female }\end{array}$ & $\begin{array}{c}\text { Juvenile } \\
\text { male }\end{array}$ & $\begin{array}{c}\text { Juvenile } \\
\text { female }\end{array}$ & $\begin{array}{c}\text { Juveniles/ } \\
\text { Adult female }\end{array}$ & $\begin{array}{c}\text { Post-breeding-season } \\
\text { age ratio (95\% CI) }\end{array}$ \\
\hline $\mathbf{2 0 1 1}$ & & & & & & \\
Mist-netting & 24 & 14 & 39 & 10 & 3.50 & $3.82(1.99-7.13)$ \\
$\begin{array}{l}\text { Night-lighting } \\
\text { Population Model }\end{array}$ & 10 & 13 & 14 & 5 & 1.46 & $1.62(0.69-3.28)$ \\
\hline $\mathbf{2 0 1 2}$ & & & & & & $1.07(0.27-2.45)$ \\
Mist-netting & 35 & 25 & 41 & 16 & 2.28 & $2.37(1.43-3.73)$ \\
$\begin{array}{l}\text { Night-lighting } \\
\text { Population Model }\end{array}$ & 9 & 13 & 2 & 3 & 0.38 & $0.42(0.06-1.00)$ \\
\hline
\end{tabular}


population model, we expected post-breeding-season age ratios derived from mist-netting and night-lighting to increase from 2011 to 2012; however, they decreased in 2012 compared to 2011 . We therefore conclude that additional assessment is needed of each of these methods. Based on point estimates, neither age ratios of woodcock captured via mist-netting nor those of woodcock captured via night-lighting appeared to be a useful proxy of local woodcock production.

If woodcock post-breeding-season age ratios at the demonstration-area scale are driven primarily by local production of young, in years of high local production both mist-netting and night-lighting would produce higher age ratios compared with age ratios from years with lower local production. Furthermore, settings with higher amounts and appropriate juxtaposition of nesting, brood-rearing, and roosting cover (e.g., woodcock demonstration areas) would have higher age ratios than settings

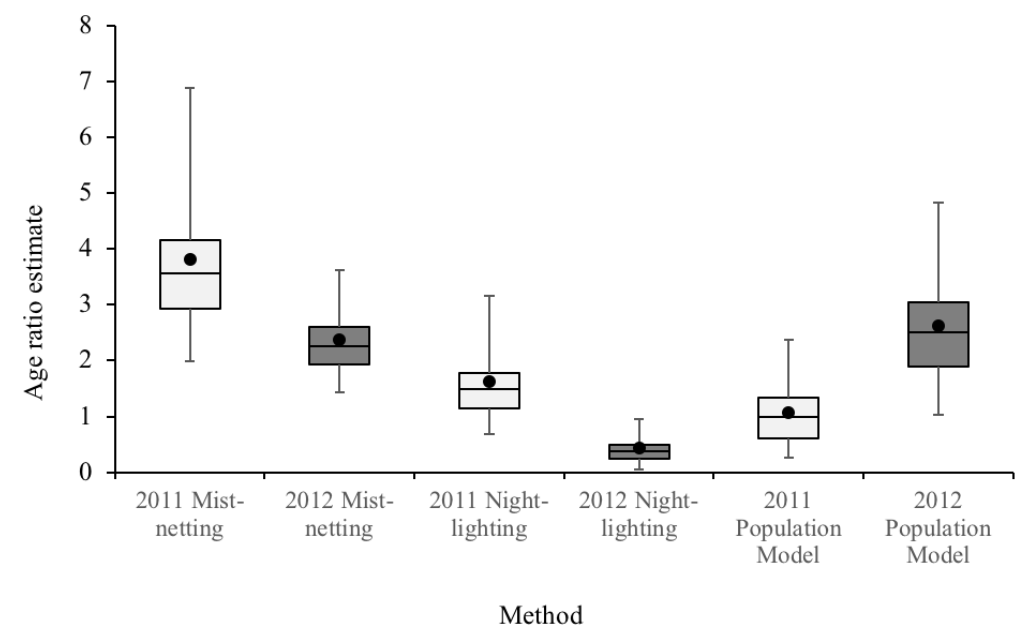

Figure 1. Post-breeding-season age ratios (juveniles/adult female) derived from capturing American woodcock in summer roosting field via mist-netting and night-lighting, and an estimate from a population model based on estimates of vital rates at Tamarac National Wildlife Refuge, Rochert, Minnesota, USA, in 2011 and 2012. Post-breedingseason age ratios from mist-netting and night-lighting were derived using bootstrapping with 1,000 iterations for 2011 and 2012. We calculated post-breeding-season age ratio for each iteration and derived the mean and $95 \%$ CI based on the distribution of post-breeding-season age ratios (excluding the lowest and highest $2.5 \%$ of estimates). Postbreeding-season age ratio estimates from our population model were informed by Kaplan-Meier survival rate estimates of adult female, nests, and juvenile woodcock. Post-breeding-season age-ratio estimates are sample means derived from bootstrapping using 1,00o iterations. Point estimates for post-breeding-season age ratios are indicated be the dot and $95 \%$ CIs are based on the distribution of the estimates. Box plot represents the quartiles of the estimates for each method by year. with other cover-type configurations (Dunford and Owen 1973). Although point estimates of age ratios of woodcock we captured via both mist-netting and night-lighting were milar between years, neither was concordant with point 2012 than 2011).

There are several possible explanations for this inconsistency. First, post-breeding-season age ratios of woodcock captured via mist-netting and night-lighting could be inflated by an influx of juvenile woodcock to areas with high-quality habitat in late summer. Second, post-breeding-season age ratios of woodcock captured via mist-netting during crepuscular periods also may be inflated (i.e., relatively higher proportion of juveniles; Table 2) if adult females are less likely to be captured in mist nets than juveniles. Capturing a relatively higher proportion of juvenile woodcock via mist-netting could be influenced by behavioral differences between juveniles and adults during crepuscular flights (Sheldon 1961, Dunford and Owen 1973, Owen and Morgan 1975). In previous studies of woodcock behavior in summer, adults flew later than juveniles; were 6 times more likely than juveniles to walk from diurnal covers to nocturnal sites, perhaps due to molt; flew shorter distances; and were less likely to take multiple flights to reach nocturnal sites (Dunford and Owen 1973, Owen and Morgan 1975). We opened mist nets prior to when woodcock began crepuscular flights and kept them open beyond when flights ceased to account for differences in flight times between juveniles and adults. Therefore, differences in flight times likely do not account for the bias toward juveniles of woodcock we captured via mist-netting. Instead, it seems that juvenile woodcock were more likely to be captured using mist nets than adults or that juveniles disproportionately immigrated into our study area in late summer. Juveniles have been observed moving greater distances than adults, especially juvenile males (Owen and Morgan 1975, Berdeen and Krementz 1998), which could lead to an influx of juveniles in summer. However, we were unable to assess this possibility in our study.

Third, how adult and juvenile woodcock use roosting fields may 
influence age ratios of woodcock captured via mist-netting and night-lighting. Although the characteristics of roosting fields used by adults and juveniles are similar (Owen and Morgan 1975, Berdeen and Krementz 1998), adult and juvenile woodcock may use these areas in different spatial and temporal patterns. For instance, Owen and Morgan (1975) reported adult woodcock remaining closer to the edge of roost fields, whereas juveniles were generally farther from edges. Moreover, Krohn (1971) and Whitcomb (1972) reported greater relative use of roosting fields by juvenile woodcock during the summer, which could be related to the higher relative abundance of juveniles following the breeding season for woodcock. The higher relative abundance of juveniles and potential higher relative use of roosting fields by juveniles would result in higher age ratios immediately post-breeding. Conversely, use of winter roosting fields by radio-marked woodcock did not vary by age or sex (Berdeen and Krementz 1998), suggesting that age and sex cohorts of woodcock change their roosting behaviors between summer and winter, or that the relatively higher use of roosting fields by juveniles in summer is solely due to higher relative abundance of juveniles in summer than winter. The point estimates of age ratios of woodcock captured via night-lighting were lower than those of woodcock captured via mist-netting and lower than those derived from our population model in 2012, which suggests that in 2012, adult females were more readily captured via night-lighting than juveniles, because they were either more abundant or easier to capture. This result contradicts previous studies that suggested juvenile woodcock use of roosting fields was higher than that of adults in summer months (Krohn 1971, Whitcomb 1972). Adults and juveniles likely use roosting fields differently during different seasons (Krohn 1971, Whitcomb 1972, Berdeen and Krementz 1998) and also may use roosting fields in different spatial and temporal patterns within the same site and season (Owen and Morgan 1975). Better understanding of how adults and juveniles use roosting fields during the post-breeding-season would be useful in interpreting age ratios of woodcock captured via mist-netting and night-lighting.

We also note that because we focused our night-lighting efforts on only 2 roosting fields ( 4.7 ha and 14.5 ha in size), our capture-related activities may have affected our success capturing woodcock at these locations. Woodcock exhibit high nocturnal site fidelity (Berdeen and Krementz 1998), but site fidelity could be affected by human activity (Sheldon 1961, Krohn 1971, Dunford and Owen 1973). Lower post-breeding-season age ratios would result if juvenile woodcock were more likely than adult females to abandon a roosting field in response to repeated capture efforts. Our post-breeding-season age ratios of woodcock captured via night-lighting in 2012 also could be lower because adult woodcock generally roost closer to edges, and therefore have a lower likelihood of being encountered (Owen and Morgan 1975) or were less likely to be captured because they were closer to areas where they could escape.

Finally, our population model may have produced biased estimates of post-breeding-season age ratios, although for the purposes of comparison with age ratios derived from mist-netting and night-lighting, our population model likely provided a reasonable estimate of the difference between 2011 and 2012 in production of young. We used values for some vital rates estimated in a previous study of woodcock reproduction and survival (e.g., hatch rate, renesting probability, and clutch sizes in first nests and renests; McAuley et al. 1990) in Maine because we were unable to estimate all of the parameters in our population model on our study area. For instance, because of the high likelihood that a female will renest, we assumed that all adult females renested following a failed first nesting attempt. Despite woodcock having a high renesting probability (McAuley et al. 1990), all woodcock that experience nest failure may not have renested on our study area, which would result in a positive bias in our model-derived estimates of post-breeding-season age ratios. Furthermore, the similarity of vital rates estimated elsewhere (e.g., McAuley et al. 1990) to those in our study population is unknown, and using these estimates could introduce bias in our estimates of post-breeding-season age ratios of unknown size and direction.

To more fully assess the utility of post-breeding-season age ratios of woodcock captured via mist-netting and night-lighting to represent production of young at the demonstration-area scale, it is likely necessary to better understand movement behavior of juveniles and adults and the relationship between woodcock movements and environmental conditions during late summer. Few studies have evaluated spatial-use patterns of woodcock at the landscape scale in summer following brood rearing (e.g., Dunford and Owen 1973, Owen and Morgan 1975). Movements and habitat selection of woodcock have been related to foraging quality and environmental conditions, especially soil moisture (Dunford and Morgan 1973, Doherty et al. 2010). Woodcock generally return to areas with high food availability, and food availability and forage quality are related to soil moisture (Doherty et al. 2010). Because woodcock also forage at night (Stribling and Doerr 1985), and it is likely that food availability is at a seasonal low in roosting fields during mid-summer due to high temperatures, adult woodcock may use roosting fields less than juvenile woodcock during summer, and may use forest cover at higher rates during summer than during the remainder of the year (Berdeen and Krementz 1998).

We also note that the post-breeding-season age ratio estimates derived from our population model provide insight into woodcock demography and population ecology. First, post-breeding-season age ratios derived from 
our model are primarily influenced by nest and juvenile survival rates, as has been demonstrated for some other bird species (e.g., Streby and Andersen 2011) that use young forest cover types. Adult female survival in our study was high and therefore had little influence on age ratios. Second, our estimates of post-breeding-season age ratios suggest that the demonstration area where we conducted our study was perhaps a population source. Estimates derived from our population model were generally greater (1.07 [95\% CI: $0.27-2.45$ ] in 2011 and 2.62 [ $95 \%$ CI: 1.04 - 4.95] in 2012) than indices in Minnesota derived from the wing-collection survey (1.o juveniles/adult female in both 2011 and 2012; Cooper and Rau 2012, 2013) and the Central Management Region (1.5 in 2011 and 1.66 in 2012; Cooper and Rau 2012, 2013).

However, even though 95\% CIs overlapped for most of our estimates of age ratios (excepting age ratios derived from night-lighting in 2012), our assessment of post-breeding-season age ratios of woodcock captured via mist-netting and night-lighting at a landscape scale likely are not reliable proxies for estimating post-breeding-season age ratios from locally derived vital rates. Without additional information about relative capture probabilities of adult versus juvenile woodcock, relative use of roosting areas by adult and juvenile woodcock, and landscape-level movements of woodcock during late summer and early fall, it is difficult to assess the usefulness of mist-netting and night-lighting to monitor production of young. Evaluating these factors at specific sites may not be practical, as doing so would likely involve investment of resources comparable to those required to estimate vital rates of local woodcock populations. Additionally, our population model included vital rate estimates from other studies as substitutes for estimates we were unable to derive in our study (e.g., hatch rate). Future studies that employ a similar approach to directly estimate woodcock productivity at a landscape scale may be better served by deriving all vital rates necessary to inform a population model from the study population. Furthermore, we suggest that future studies incorporate the assumptions and drawbacks of each of the methods we deployed and that further evaluation is necessary before indirect methods can be reliably used as proxies of local woodcock recruitment.

\section{Management Implications}

Surveying wildlife populations following habitat management is a critical part of adaptive wildlife management. A current strategy for increasing woodcock abundance is establishment of an abundance of cover types used by woodcock at a landscape scale, as exemplified in demonstration areas. However, it is not obvious how best to assess woodcock population response in these landscapes. Estimating productivity of young derived from local vital rates is time consuming and expensive, but presumably provides productivity estimates that best reflect local populations. Age ratios of woodcock captured via mist-netting and night-lighting require less time and expense to obtain, but additional assessment of these methods is needed prior to knowing if they are useful proxies of estimating local productivity. In our assessment at a woodcock demonstration area in west-central Minnesota, USA, neither point estimates of age ratios of woodcock captured via mist-netting nor of woodcock captured via night-lighting seemed to reflect the difference between years we observed in age-ratio estimates from our population model, which incorporated direct estimates of production of young. This suggests that other factors (e.g., influx of juvenile woodcock into areas with high habitat quality) may be influencing post-breeding-season age ratios derived from mist-netting and night-lighting. Future studies designed to evaluate local woodcock production may benefit from further assessment of the methods used and evaluation of the assumptions inherent in each method.

\section{Acknowledgments}

We thank J. Nelson, M. Johnson, D. Page, and J. Lehman for their long hours assisting in capturing, radio marking, and tracking woodcock. We especially are grateful to D. Dustin and E. Johnson for volunteering many hours running dogs through the woods to find woodcock nests and broods, D. McAuley for his valuable insights on capture methods, and H. Streby, J. Fieberg, and T. Arnold for assistance with analytical techniques. We thank Tamarac National Wildlife Refuge staff for access to refuge lands, equipment, lodging, and logistical support. Federal and State permits for capture, banding, and radio-marking were granted by the U.S. Geological Survey Bird Banding Laboratory (Permit no. 06258, Wayne Brininger) and the Minnesota Department of Natural Resources (Permit nos. 17377 and 17973, Wayne Brininger). Use of trade, firm, or product names does not imply endorsement by the U.S. Government or the University of Minnesota. The findings and conclusions in this article are those of the author(s) and do not necessarily represent the views of the U.S. Fish and Wildlife Service. The authors of this manuscript report that they have no potential sources of conflict of interest that might be perceived as influencing the authors' objectivity. This project was funded by the U.S. Fish and Wildlife Service Webless Migratory Game Program through Research Work Order no. 91 at the U.S. Geological Survey Minnesota Cooperative Fish and Wildlife Research Unit, with additional funding provided by Woodcock Minnesota.

\section{Literature Cited}

Ammann, G.A. 1974. Methods of capturing American woodcock broods. Pages 593-605 in S. Lundstrom, editor. Eleventh International Congress of Game Biologists. Stockholm, Sweden. 
Ammann, G.A. 1977. Finding and banding woodcock broods using pointing dogs. Michigan Department of Natural Resources, Wildlife Division Report 2780.

Berdeen, J.B., and D.G. Krementz. 1998. The use of fields at night by wintering American woodcock. Journal of Wildlife Management 62:939-947.

Cooper, T.R., and R. Rau. 2012. American woodcock population status, 2012. U.S. Fish and Wildlife Service, Office of Migratory Bird Management, Laurel, Maryland, USA.

Cooper.T.R., and R. Rau. 2013. American woodcock population status, 2013. U.S. Fish and Wildlife Service, Office of Migratory Bird Management, Laurel, Maryland, USA.

Daly, K.O., D.E. Andersen, W.L. Brininger, and T.R. Cooper. 2015 Radio-transmitters have no impact on survival of pre-fledged American woodcocks. Journal of Field Ornithology 86:345-351.

Doherty, K.E., D.E. Andersen, J. Meunier, E. Oppelt, R.S. Lutz, and J.G. Bruggink. 2010. Foraging location quality as a predictor of fidelity to a diurnal site for adult female American woodcock Scolpax minor. Wildlife Biology 16:379-388.

Dunford, R.D., and R.B. Owen, Jr. 1973. Summer behavior of immature radio-equipped woodcock in central Maine. Journal of Wildlife Management 37:462-469.

Dwyer, T.J., G.F. Sepik, E.L. Derleth, and D.G. McAuley. 1988. Demographic characteristics of a Maine woodcock population and effects of habitat management. U.S. Fish and Wildlife Service, Fish Wildlife Resource Report 4, Washington D.C., USA.

Gregg, L. 1984. Population ecology of woodcock in Wisconsin. Wisconsin Department of Natural Resources, Technical Bulletin 144 Madison, Wisconsin, USA.

Hale, J.B., and L.E. Gregg. 1976. Woodcock use of clearcut aspen areas in Wisconsin. Wildlife Society Bulletin 4: 111-115.

Kelley, J.R., S.J. Williamson, and T.R. Cooper (editors). 2008. American Woodcock Conservation Plan: a summary of and recommendations for woodcock conservation in North America. Wildlife Management Institute, Washington, D.C., USA.

Korschgen, C.E., K.P. Kenow, W.L. Green, D.H. Johnson, M.D. Samuel, L. Sileo. 1996. Survival of radiomarked canvasback ducklings in northwestern Minnesota. Journal of Wildlife Management 6o:120-132.

Krohn, W.B. 1971. Some patterns of woodcock activity on Maine summer fields. Wilson Bulletin 83:396-407.

Martin, F.W. 1964. Woodcock age and sex determination from wings. Journal of Wildlife Management 28:287-293.

McAuley, D.G., J.R. Longcore, D.A. Clugston, R.B. Allen, A. Weik, S. Williamson, J. Dunn, B. Palmer, K. Evans, W. Staats, G.F. Sepik, and W. Halteman. 2005. Effects of hunting on survival of American woodcock in the Northeast. Journal of Wildlife Management 69:1565-1577.

McAuley, D.G., J.R. Longcore, and G.F. Sepik. 1990. Renesting by American woodcocks (Scolopax minor) in Maine. Auk 107:407-410.

McAuley, D.G., J.R. Longcore, and G.F. Sepik. 1993a. Methods of woodcock research: experiences and recommendations. Pages
5-11 in J.R. Longcore and G.F. Sepik, editors. Eighth American Woodcock Symposium. U.S. Fish and Wildlife Service, Wildlife Resource Report 16, Washington D.C., USA

McAuley, D.G., J.R. Longcore, and G.F. Sepik. 1993b. Behavior of radio-marked breeding American woodcocks. Pages 116-125 in J.R. Longcore and G.F. Sepik, editors. Eighth American Woodcock Symposium. U.S. Fish and Wildlife Service, Wildlife Resource Report 16, Washington D.C., USA.

Mendall, H.L. 1938. A technique for banding woodcock. Bird-Banding 9:153-155.

Nur, N., A.L. Holmes, and G.R. Geupel. 2004. Use of survival time analysis to analyze nesting success in birds: an example using loggerhead shrikes. Condor 106:457-471.

Owen, R.B., Jr., and J.W. Morgan. 1975. Summer behavior of adult radio-equipped woodcock in central Maine. Journal of Wildlife Management 39:179-182.

Pollock, K.H., S.R. Winterstein, C.M. Bunck, and P.D. Curtis. 1989. Survival analysis in telemetry studies: the staggered entry design. Journal of Wildlife Management 53:7-15.

Sauer, J.R., and J.B. Bortner. 1991. Population trends from the American Woodcock Singing-ground Survey, 1970-88. Journal of Wildlife Management 55:300-312.

Seamans, M.E., and R.D. Rau. 2017. American woodcock population status, 2017. U.S. Fish and Wildlife Service, Laurel, Maryland, USA.

Sepik, G.F. 1994. A woodcock in the hand. Ruffed Grouse Society, Coraopolis, Pennsylvania, USA.

Sheldon, W.G. 1961. Summer crepuscular flights of American woodcock in central Massachusetts. Wilson Bulletin 73:126-139.

Sheldon, W.G. 1971. The book of the American woodcock. University of Massachusetts Press, Amherst, Massachusetts, USA.

Streby, H.M., and D.E. Andersen. 2011. Seasonal productivity in a population of migratory songbirds: why nest data are not enough. Ecosphere 2: Article 78.

Stribling, H.L., and P.D. Doerr. 1985. Nocturnal use of fields by American woodcock. Journal of Wildlife Management 49:485-491.

Winterstein, S.R. 1992. Chi-square tests for intrabrood independence when using the Mayfield Method. Journal of Wildlife Management 58:398-401.

Whitcomb, D.A. 1972. Nocturnal use of forest clearings during summer by an insular woodcock population. Thesis, Michigan State University, East Lansing, USA.

Wildlife Management Institute. 2009. Best management practices for woodcock and associated bird species: Upper Great Lakes Woodcock and Young Forest Initiative. Wildlife Management Institute. $<$ https://youngforest.org/resource/best-management-practices-woodcock-associated-bird-species-upper-great-lakes-woodcock-and $>$ Accessed 7 September 2017.

Wildlife Management Institute. 2010. Implementing the American Woodcock Conservation Plan: progress to date. Wildlife Management Institute, Washington D.C., USA. 